\section{NEONATAL MORTALITY IN VERY-LOW- BIRTHWEIGH INFANTS (VLBWI) FROM TWO NEONATAL NETWORKS IN SPAIN AND JAPAN}

M. Madrid ${ }^{1}$, A. Azpeitia ${ }^{1}$, A. Valls i Soler ${ }^{1}$, R. Mori ${ }^{2}$

${ }^{1}$ Neonatal Epidemiology Unit, Hospital Cruces, University Basque Country, Bilbao, Spain, ${ }^{2}$ Department of Global Health Policy, University of Tokyo, Tokyo, Japan

Background: VLBWI mortality rates vary across NICUs, however few comparisons are available between different national networks.

Aim: To compare and analyze differences in neonatal mortality rates of two VLBWI cohorts from Neonatal Networks in Spain and Japan.

Methods: Risk/protective factors, complications, and morbi-mortality outcomes were compared among inborn VLBWI admitted to NICU's from SEN-1500 (7,006 infants, 63 NICU's) and NRNJ (8,695 infants, 69 NICU's) from 2005 to 2007. Nonparametric independent comparison analysis and logistic regression models were performed to predict mortality adjusting for perinatal risks factors.

Results: Significant differences were found in gestation [mean(SD); 29.3(2.9) vs. 28.6(3.3) wks] and birthweigh [1117(271) vs. 1031(304)g. Both, 28-days neonatal and pre-discharge mortality rates were significantly higher in SEN-1500 $(12.5 \%$ and $14.7 \%$ ) than in NRNJ (6.4 and 9.2\%). Differences in mortality remained even when adjusting for GA, birthweight, prenatal steroid use, 1 and 5 -min Apgar scores and presence of major congenital anomalies. There were many differences in clinical management among networks. In SEN-1500, use of surfactant and high frequency ventilation and DBP and PDA ligation rates were lower, but had higher rates of NEC, grades ${ }_{3-4} \mathrm{IVH}$, and specially of sepsis $(32.7 \%$ vs. $7.3 \%$; $<<0.001)$.

Conclusion: Differences in neonatal and predischarge mortality rates of VLBWI form SEN-1500 and NRNJ could not completely be explained by initial risk and protective factors. Sepsis-related mortality accounted for $61.3 \%$ of the excess mortality in SEN-1500. The implementation of systematic preventive strategies for sepsis seems mandatory.

Acknowledgements: Patients and participanting NICU's from both SEN-1500 and NRNJ Networks.

\section{HOW USEFUL ARE BLOOD CULTURES ON PICU?}

\author{
B. Lakin, J. Sharp, F. Potter
}

PICU, Royal Liverpool Childrens NHS Foundation Trust, Liverpool, UK

Objectives: To determine the current practice of obtaining blood cultures sent from PICU.

Methods: Retrospective study of all blood cultures sent from patients in one calendar month (January 2009).

Results: A total of 140 blood cultures were sent from 34 patients over 89 screening episodes. Only $22 \%$ of screening episodes achieved the minimum requirement of cultures of blood, urine and ET aspirates. Neither clinical parameters nor infective indices showed reliable correlation with positive blood cultures. $23(16 \%)$ of blood cultures were positive whilst 9 samples were attributed to probable contaminants. A single patient (with Candidaemia) accounted for 10 positive samples. Blood cultures were obtained from different sites including peripheral (36), arterial (54), central (50). The number of positive cultures were equivalent from each of these sites. Of the patients screened after admission 60 were already receiving IV antibiotics.

Conclusions: Blood cultures were commonly performed and demonstrated a low overall yield perhaps as a result of the criteria used for screening. Several areas of practice warrant improvement including obtaining a complete screen more often and implementing measures to reduce the contamination of peripheral samples (no touch technique).A prospective study would be needed to determine the criteria for screening more accurately. Furthermore, there is a need for more reliable indices of infection which may also reduce the use of antimicrobials.

1392

\section{MONITORING OF SERUM VANCOMYCIN LEVELS IN ICU PEDIATRIC PATIENTS}

\author{
M. Kazantzi, D. Paraschou, P. Kalabalikis, \\ T. Hatzis \\ Pediatric Intensive Care Unit, Agia Sofia Children's \\ Hospital, Athens, Greece
}

Aims: To analyze the results from the clinical monitoring of serum vancomycin levels among ICU pediatric patients. 